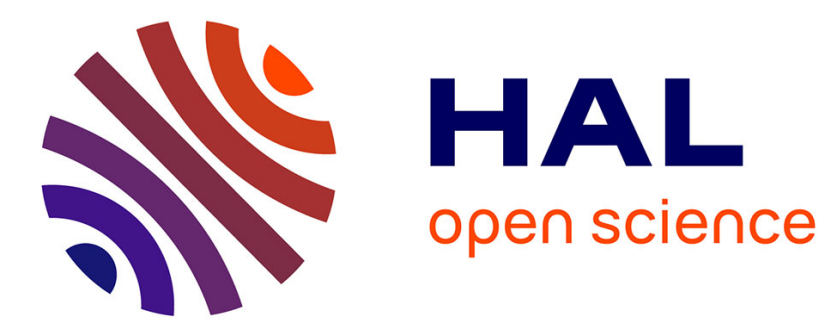

\title{
Shape Optimization Problem for Heat Equation
}

Antoine Henrot, Jan Sokolowski

\section{To cite this version:}

Antoine Henrot, Jan Sokolowski. Shape Optimization Problem for Heat Equation. [Research Report] RR-3185, INRIA. 1997, pp.23. inria-00073504

\section{HAL Id: inria-00073504 https://hal.inria.fr/inria-00073504}

Submitted on 24 May 2006

HAL is a multi-disciplinary open access archive for the deposit and dissemination of scientific research documents, whether they are published or not. The documents may come from teaching and research institutions in France or abroad, or from public or private research centers.
L'archive ouverte pluridisciplinaire HAL, est destinée au dépôt et à la diffusion de documents scientifiques de niveau recherche, publiés ou non, émanant des établissements d'enseignement et de recherche français ou étrangers, des laboratoires publics ou privés. 
INSTITUT NATIONAL DE RECHERCHE EN INFORMATIQUE ET EN AUTOMATIQUE

\section{Shape Optimization Problem for Heat Equation}

Antoine Henrot and Jan Sokołowski

\section{$\mathbf{N}^{\circ} 3185$}

Juin 1997

THÈME 4 



\title{
Shape Optimization Problem for Heat Equation
}

\author{
Antoine Henrot ${ }^{*}$ and Jan Sokołowski ${ }^{\dagger}$ \\ Thème 4 - Simulation et optimisation \\ de systèmes complexes \\ Projet Numath
}

Rapport de recherche $\mathrm{n}^{\circ} 3185$ - Juin 1997 - 23 pages

\begin{abstract}
In this paper the support of a Radon measure is selected in an optimal way. The solution of the parabolic equation depends on the mesure via the mixed type boundary conditions. The existence of a solution for a class of domain optimization problems is shown. We also investigate the behaviour of the optimal solution for some time $T$, when $T \rightarrow \infty$ and we prove that it converges to the optimal solution of the stationary problem. The first order necessary optimality conditions are derived.
\end{abstract}

Key-words: shape optimization, Radon measure, shape derivative, parabolic equation

(Résumé : tsvp)

* Equipe de Mathématique, UMR CNRS, Université de Franche-Comté, 25030 Besancon Cedex, France; e-mail: henrot@math.univ-fcomte.fr

$\dagger$ Institut Elie Cartan, Laboratoire de Mathématiques, Université Henri Poincaré Nancy I, B.P. 239, 54506 Vandoeuvre lès Nancy Cedex, France and Systems Research Institute of the Polish Academy of Sciences, ul. Newelska 6, 01-447 Warszawa, Poland; e-mail: sokolows@iecn.u-nancy.fr 


\section{Problème d'optimisation de forme pour l'equation de la chaleur}

Résumé : Dans cet article, on cherche à sélectionner une courbe optimale représentant une source de chaleur. Cette courbe apparait comme le support d'une mesure intervenant dans l'équation de la chaleur par l'intermédiaire d'une condition au bord. On montre l'existence d'une solution optimale dans une classe raisonnable de courbes admissibles. On s'intéresse également au comportement de cette solution optimale pour un temps $T$ quand on fait tendre $T$ vers l'infini et on montre qu'elle converge vers la solution optimale du problème stationnaire. On explicite également les conditions d'optimalité du premier ordre.

Mots-clé : optimisation de forme, mesure de Radon, la dérivée par rapport au domaine, equation parabolique 


\section{Introduction}

In this paper we will consider a problem related to the following. Given a flat piece of material - a pane of glass in a window for example - we attach a heating wire to one surface of this material. This wire is modelled as a continuous curve connecting to fixed points $A$ and $B$. We want to investigate which curve would optimize the temperature distribution on the opposite surface at a given time?

We refer the reader to (Henrot, Horn and Sokolowski, 1996) for the related results in the stationary case. In the paper the time dependent problem is considered. We prove, under appropriate assumptions on the set of admissible curves, the existence of an optimal solution. We also investigate the behaviour of the optimal solution for $T$, when $T \rightarrow \infty$ and we prove that it converges to the optimal solution of the stationary problem. The first order necessary optimality conditions are derived.

\section{Existence of a classical solution}

\subsection{Presentation of the problem}

We assume that $\Omega$ is a simply connected domain in $\mathbb{R}^{2}$ and let $\Sigma=\Omega \times(0, d)$. We denote $\Omega_{0}=\Omega \times\{0\}, \Omega_{1}=\Omega \times\{d\}$ and $\Gamma=\partial \Omega \times(0, d)$. Therefore

$$
\partial \Sigma=\Omega_{0} \cup \Omega_{1} \cup \Gamma .
$$

Given a curve $\gamma \subset \Omega_{0}$ parametrized by $\ell \in[0,1]$, we assume that $A=\gamma(0)$ and $B=\gamma(1)$ are fixed points in $\Omega_{0}$. We are interested in the heat equation where $\gamma$ can be looked as the heat source. For physical reasons, it seems reasonable to consider such a heat source independent of the time $t$. So let us consider the following problem where $u=u(x, t)$ is the temperature.

$\left(\mathcal{P}_{1}(\gamma)\right)$

$$
\left\{\begin{array}{rlrl}
\frac{\partial u}{\partial t}-\Delta u & =0 & & \text { in } \Sigma \times(0, T), \\
-\frac{\partial u}{\partial n}=\kappa u & & \text { on } \Gamma \times(0, T)
\end{array}\right.
$$

RR $n^{\circ} 3185$ 


$$
\left\{\begin{array}{rlrl}
-\frac{\partial u}{\partial n} & =u-\varphi_{1} & \text { on } \Omega_{1} \times(0, T) \\
-\frac{\partial u}{\partial n} & =u-\varphi_{0}-\delta_{\gamma} & & \text { on } \Omega_{0} \times(0, T) \\
u(x, 0) & = & u_{0}(x) & \text { in } \Sigma,
\end{array}\right.
$$

where $\kappa \geq 0, \varphi_{0}, \varphi_{1}$ are given $L^{2}$ functions (independent of $t$ ), $\delta_{\gamma}$ is a Dirac measure supported on the curve $\gamma$, and the initial data belongs to $L^{2}(\Sigma)$. We denote by $\mathcal{P}_{1}(\mu)$ the initial boundary value problem with the Dirac measure $\delta_{\gamma}$ replaced by a Radon measure $\mu$.

Let us introduce the Banach spaces $V=W^{1, p}(\Sigma), \frac{6}{5} \leq p<\frac{3}{2}$ (therefore, by the Sobolev-Rellich theorem $V \subset L^{2}(\Sigma)$ with compact imbedding), $H=L^{2}(\Sigma)$ and $V^{\prime}$ the dual space of $V$ and let us denote by

$$
W(0, T)=\left\{u \in L^{2}(0, T ; V) \quad \text { such that } \frac{\partial u}{\partial t} \text { belongs to } L^{2}\left(0, T ; V^{\prime}\right)\right\} .
$$

In section 2.4 the space $W_{s}(0, T)$ is considered for $V=W^{1, p}\left(\Sigma_{s}\right)$, where $\Sigma_{s}=$ $T_{s}(\Sigma)$ is introduced in section 2.4 .

By classical results, we refer to (Lions, Magenes, 1968) and (Aubin, 1963) the space $W(0, T)$ is continuously embedded in $C(0, T ; H)$ the space of continuous functions from $[0, T]$ in $H$ and compactly embedded in $L^{2}\left(0, T ; L^{2}(\Sigma)\right)$.

Since the problem involves a Radon measure on a part of the boundary, we need to define in a convenient way the notion of a solution to the parabolic problem $\mathcal{P}_{1}(\gamma)$. Since, in our case the measure does not depend $t$, we have the following representation of solutions to $\mathcal{P}_{1}(\gamma)$.

Remark 2.1 The solution to the problem $\mathcal{P}_{1}(\gamma)$ is of the form

$$
u(x, t)=\bar{u}(x, t)+w(x),
$$

where $w \in W^{1, p}(\Sigma)$ is the unique solution to the stationary problem

$$
\left\{\begin{array}{rlrl}
-\Delta w & =0 & \text { in } \Sigma, \\
-\frac{\partial w}{\partial n}=\kappa w & \text { on } \Gamma,
\end{array}\right.
$$




$$
\left\{\begin{array}{rlr}
-\frac{\partial w}{\partial n} & =w-\varphi_{1} & \text { on } \Omega_{1} \\
-\frac{\partial w}{\partial n} & =w-\varphi_{0}-\delta_{\gamma} & \text { on } \Omega_{0}
\end{array}\right.
$$

and $\bar{u}(x, t)$ satisfies the following parabolic equation

$$
\left\{\begin{array}{rlrl}
\frac{\partial \bar{u}}{\partial t}-\Delta \bar{u} & =0 & & \text { in } \Sigma \times(0, T), \\
-\frac{\partial \bar{u}}{\partial n} & =\kappa \bar{u} & & \text { on } \Gamma \times(0, T), \\
-\frac{\partial \bar{u}}{\partial n} & =\bar{u} & & \text { on } \Omega_{1} \times(0, T), \\
-\frac{\partial \bar{u}}{\partial n} & =\bar{u} & \text { on } \Omega_{0} \times(0, T), \\
\bar{u}(x, 0) & =u_{0}(x)-w(x) & \text { in } \Sigma
\end{array}\right.
$$

so, it is enough to define the solution in the stationary case.

The stationary case is considered in (Henrot, Horn and Sokolowski, 1996) in a classical way using the duality method. We recall the result here, applied to our problem.

Proposition 2.1 Let $\mu$ be a bounded Radon mesure supported on $\Omega_{0}$. There exists the unique solution $w \in W^{1, p}(\Sigma)$, for all $p \in\left[1, \frac{3}{2}\right)$, to the problem

$$
\left\{\begin{array}{rccc}
-\Delta w & = & 0 & \text { in } \Sigma, \\
-\frac{\partial w}{\partial n} & = & \kappa w & \text { on } \Gamma, \\
-\frac{\partial w}{\partial n} & = & w-\varphi_{1} & \text { on } \Omega_{1}, \\
-\frac{\partial w}{\partial n}= & w-\varphi_{0}-\mu & \text { on } \Omega_{0},
\end{array}\right.
$$

RR $n^{\circ} 3185$ 
moreover, there exists constants $C_{1}$ and $C_{2}$ depending only on $\Sigma, \varphi_{0}$ and $\varphi_{1}$ such that

$$
\|w\|_{W^{1, p}(\Sigma)} \leq C_{1}+C_{2}\|\mu\|_{\mathcal{M}_{b}\left(\Omega_{0}\right)} .
$$

Using this result, the "decoupling" remark 2.1 and classical estimates for the parabolic equation satisfied by $\bar{u}$ we obtain immediately that $u=\bar{u}+w$ belongs to the space $W(0, T)$ and

$$
\|u\|_{W(0, T)} \leq C_{1}+C_{2}\|\mu\|_{\mathcal{M}_{b}\left(\Omega_{0}\right)} .
$$

Moreover, it is a consequence of the above results that the variational formulation of the heat equation $\mathcal{P}_{1}(\gamma)$ is given as follows.

Find $u \in W(0, T)$ such that for all functions $v \in W^{1, q}(\Sigma)$

$\left(\mathcal{P}_{2}(\gamma)\right)$

$$
\left\{\begin{array}{l}
\frac{d}{d t}(u(t), v)+a(u(t), v)=L(v) \\
u(0)=u_{0}
\end{array}\right.
$$

in the sense of distributions on $(0, T)$, where

$$
\begin{aligned}
a(u, v) & =\int_{\Sigma} \nabla u \cdot \nabla v d x+\int_{\Omega_{1}} u v d \sigma+\int_{\Omega_{0}} u v d \sigma+\kappa \int_{\Gamma} u v d \sigma, \\
L(v) & =\int_{\Omega_{1}} \varphi_{1} v d \sigma+\int_{\Omega_{0}} \varphi_{0} v d \sigma+<\delta_{\gamma}, v>, \\
(u, v) & =\int_{\Sigma} u v d x .
\end{aligned}
$$

From the inequality (1) and the compact embedding of $W(0, T)$ in $L^{2}\left(0, T ; L^{2}(\Sigma)\right)$ we obtain the following continuity result of solutions to $\mathcal{P}_{1}$ with $\delta_{\gamma}$ replaced by a mesure $\mu$ with respect to the measure $\mu$.

Proposition 2.2 Given a sequence $\left\{\mu_{n}\right\}$ of Radon measures supported on $\Omega_{0}$, $\left\|\mu_{n}\right\|_{\mathcal{M}_{b}\left(\Omega_{0}\right)} \leq C$, there exists a subsequence, still denoted by $\left\{\mu_{n}\right\}$ and a Radon measure $\mu \in \mathcal{M}_{b}\left(\Omega_{0}\right)$ such that

$$
\begin{array}{r}
\mu_{n} \rightarrow \mu \quad \text { in } \mathcal{M}_{b}\left(\Omega_{0}\right) \text { weak }-(*), \\
u_{n} \rightarrow u \quad \text { strongly in } L^{2}\left(0, T ; L^{2}(\Sigma)\right) \text { and weakly in } L^{2}(0, T ; V), \\
\frac{d u_{n}}{d t} \rightarrow \frac{d u}{d t} \quad \text { weakly in } L^{2}\left(0, T ; V^{\prime}\right) .
\end{array}
$$

INRIA 
where $u_{n}, n=1,2, \ldots$, is a solution to $\mathcal{P}_{1}\left(\mu_{n}\right)$.

Proof. From the boundedness assumption of the sequence $\mu_{n}$, we have immediately, in view of (1) that $u_{n}$ is bounded in $W(0, T)$ and then converges strongly in $L^{2}\left(0, T ; L^{2}(\Sigma)\right)$ and weakly in $W(0, T)$ to a function $u^{*}$. The only point that remains to be proved is that $u^{*}$ is the solution to the parabolic problem $\mathcal{P}_{1}(\mu)$ for the weak- $(*)$ limit $\mu$ of the sequence $\mu_{n}$.

Using the elliptic equation in Proposition 2.1 with $\mu$ replaced by $\mu_{n}$ we have the weak convergence of the sequence of solutions $w_{n} \in W^{1, p}(\Sigma)$ to the limit $w^{*}$. Then, for the initial condition $\bar{u}_{n}(x, 0)=u_{0}(x)-w_{n}(x)$ the sequence of solutions $\bar{u}_{n}$ to the parabolic system in remark 2.1 converges weakly in the space $W(0, T)$ to the solution $\bar{u}^{*}$. Using the remark 2.1 it follows that $u^{*}=\bar{u}^{*}+w^{*}$ is a solution to $\mathcal{P}(\mu)$.

Since the solution to the problem $\mathcal{P}(\mu)$ is unique, it follows that $u^{*}=u$. Now, since $u$ is the unique accumulation point of the sequence $u_{n}$, the whole sequence converges to $u$, which completes the proof.

Remark 2.2 In order to show that $u_{n} \rightarrow u$ strongly in $L^{2}\left(0, T ; H^{1}(\Sigma)\right)$ it is sufficient to have the following convergence

$$
<\mu_{n}, u_{n}>\rightarrow<\mu, u>\text {. }
$$

Indeed, using (4) and classical arguments, we have

$$
X_{n}(T):=\frac{1}{2}\left|u_{n}(T)-u(T)\right|^{2}+\int_{0}^{T} a\left(u_{n}(t)-u(t), u_{n}(t)-u(t)\right) d t \longrightarrow 0
$$

furthermore

$$
0 \leq \alpha \int_{0}^{T}\left\|u_{n}(t)-u(t)\right\|^{2} d t \leq X_{n}(T)
$$

therefore, the strong convergence follows.

Lemma 2.3 The sequence $u_{n}(T, x)$ converges weakly to $u(T, x)$ in $L^{2}(\Sigma)$ for any fixed $0<T<\infty$.

Proof. Let us take $v=u_{n}$ in the variational formulation, the bilinear form $a(\cdot, \cdot)$ being coercive by the Friedrichs-Poincaré inequality, by integrating the resulting inequality over $(0, T)$ we obtain,

$$
\frac{1}{2}\left|u_{n}(T)\right|_{H}^{2}+\alpha \int_{0}^{T}\left\|u_{n}\right\|_{V}^{2} \leq C\left\|u_{n}\right\|_{V}+\frac{1}{2}\left|u_{0}\right|_{H}^{2}
$$

RR $n^{\circ} 3185$ 
Therefore, the sequence $u_{n}(T, x)$ is bounded in $L^{2}(\Sigma)$, so we can extract a subsequence which converges weakly. In the same way as before we have proved that $u^{*}(0)=u_{0}$, we are able to show that the weak limit of $u_{n}(T)$ is necessarily $u(T)$, and since this limit is unique, the whole sequence $u_{n}(T)$ converges weakly to $u(T)$.

\subsection{Admissible curves}

We are going to define the set of admissible curves $\gamma$. Any admissible curve $\gamma$ is the support of the Radon measure which is the heat source for the problem under considerations.

To this end we denote by $Q$ the cube $Q=(0,1) \times(0,1)$, by $I \subset Q$ the interval $I=\left[-\frac{1}{2}, \frac{1}{2}\right] \times\{0\}$.

Definition 2.1 A given curve $\gamma$ is called admissible if there exists a one-toone mapping $F: Q \mapsto \mathcal{O}$, where $\mathcal{O}$ denotes an open neighbourhood of $\gamma$ in $\Omega_{0}$ such that

$$
\begin{array}{rl}
F(Q)=\mathcal{O} & F(I)=\gamma \\
\|F\|_{W^{1, \infty}(Q)} \leq L_{1} & \left\|F^{-1}\right\|_{W^{1, \infty}(\mathcal{O})} \leq L_{2}
\end{array}
$$

Prescribing uniform bounds $L=L_{1}=L_{2}>0$ and assuming that the following compactness condition is satisfied

$(\mathcal{H})$ Given a sequence $F_{n}$ which satisfies uniformly the latter bounds, there exists a subsequence, still denoted by $F_{n}$ such that

$$
\left|F_{n}^{\prime}(\cdot, 0)\right| \rightarrow\left|F^{\prime}(\cdot, 0)\right| \quad \text { weakly in } L^{2}\left(-\frac{1}{2}, \frac{1}{2}\right) .
$$

we define an admissible family

$\mathcal{F}_{L}=\left\{\gamma\right.$ is admissible $\mid(\mathcal{H})$ is satisfied, $\|F\|_{W^{1, \infty}(Q)} \leq L$ and $\left.\left\|F^{-1}\right\|_{W^{1, \infty}(\mathcal{O})} \leq L\right\}$

where $L>0$ is a given constant. 
Remark 2.3 Without the assumption $(\mathcal{H})$ on the family $\mathcal{F}_{L}$ we cannot expect that for any sequence $\left\{\gamma_{n}\right\} \subset \mathcal{F}_{L}$, there exists a subsequence, still denoted by $\left\{\gamma_{n}\right\}$ such that

$$
\delta_{\gamma_{n}} \rightarrow \delta_{\gamma} \quad \text { weak }-(*) \text { in the space } \mathcal{M}_{b}\left(\Omega_{0}\right) \text {. }
$$

A counterexample can be constructed using $F_{n}(x, y)=\left\{x, y+\frac{1}{n} \sin (n x)\right\}$.

Remark 2.4 We use the above definition of a set of admissible curves $\mathcal{F}_{L}$, since we want to apply an appropriate trace theorem on $\gamma$. Such a definition is better suited for our applications than the simple definition of curves parametrized over an interval.

Remark 2.5 We can replace definition 2.1 by a more general notion of a Lipschitzian manifold, where the existence of a global parametrization is not required. We prefer to work with the global parametrization for the sake of simplicity. The same result can be obtained for the more general setting of a Lipschitzian manifold, provided that the uniform bounds are prescribed with the same Lipschitz constant for any collection of charts. Using a partition of unity the problem can be localized in a standard way.

Remark 2.6 Some classes of admissible curves in the plane are introduced by I.I. Daniliuk (Daniliuk, 1975) in the framework of integral equations in non-smooth domains.

On the other hand, it seems to be possible to use some families of admissible curves defined by using capacity type constraints, which probably assure the existence of a solution in a slightly wider class. But this approach is rather complicated and it is not evident that such families of admissible curves can be of any interest for the numerical methods. We refer the reader to the monograph (Ziemer, 1989) for the definition and properties of capacity, and to (Bucur, Zolesio, 1995) for some results in the case of admissible domains with capacitary constraints for homogeneous Dirichlet problems.

An admissible curve is defined in the parametric form

$$
\left\{\begin{array}{l}
x(\ell)=F_{1}(\ell, 0) \\
y(\ell)=F_{2}(\ell, 0)
\end{array} \quad \ell \in\left[-\frac{1}{2}, \frac{1}{2}\right]\right.
$$

RR $n^{\circ} 3185$ 
where $F=\left(F_{1}, F_{2}\right)$ is bi-Lipschitz mapping. For $\gamma \in \mathcal{F}_{L}$ it follows that

$$
P(\gamma)=\int_{-\frac{1}{2}}^{\frac{1}{2}} \sqrt{x^{\prime 2}(\ell)+y^{\prime 2}(\ell)} d \ell=\int_{-\frac{1}{2}}^{\frac{1}{2}}\left(\frac{\partial F_{1}{ }^{2}}{\partial x}(\ell, 0)+{\frac{\partial F_{2}}{\partial x}}^{2}(\ell, 0)\right)^{\frac{1}{2}} d \ell \leq \sqrt{2} L
$$

therefore the length of admissible curves in the set $\mathcal{F}_{L}$ is uniformly bounded, but the uniform boundedness of the length is a weaker condition for a curve than the condition to be a member of $\mathcal{F}_{L}$.

Proposition 2.4 Given a sequence of curves $\gamma_{n}$ in $\mathcal{F}_{L}$, there exists a curve $\gamma \in \mathcal{F}_{L}$ and a subsequence $\gamma_{n_{k}}$ such that

$$
\delta_{\gamma_{n_{k}}} \rightarrow \delta_{\gamma} \quad \text { weak-(*) in the space } \mathcal{M}_{b}\left(\Omega_{0}\right) \text {. }
$$

Proof. Given $\gamma_{n}=F_{n}\left(Q_{0}\right) \in \mathcal{F}_{L}$, we have

$$
\left\|F_{n}\right\|_{W^{1, \infty}} \leq L \quad \text { and } \quad\left\|F_{n}^{-1}\right\|_{W^{1, \infty}} \leq L
$$

By the theorem of Ascoli there exists a function $F$ which is continuous over $Q$ such that for a subsequence $F_{n_{k}}$

$$
F_{n_{k}}(x) \rightarrow F(x) \quad \text { uniformly over } \bar{Q} \text {. }
$$

The functions $F_{n_{k}}$ are uniformly Lipschitz continuous with the constant $L$, the same remains valid for $F$, thus $F \in W^{1, \infty}(Q)$ with $\|F\|_{W^{1, \infty}} \leq L$. We denote $\gamma=F\left(Q_{0}\right)$.

Furthermore, the inequality $\left\|F_{n}^{-1}\right\|_{W^{1, \infty}} \leq L$ implies that

$$
\left|F_{n}(x)-F_{n}(y)\right| \geq \frac{1}{L}|x-y| \quad \forall x, y \in Q
$$

hence taking the limit it follows that

$$
|F(x)-F(y)| \geq \frac{1}{L}|x-y| \quad \forall x, y \in Q
$$

which shows that $F$ is one-to-one. We denote $\mathcal{O}=F(Q)$, thus there exists the inverse mapping $F^{-1}: \mathcal{O} \mapsto Q, F^{-1}$ being Lipschitz continuous with the constant $L$ in view of the latter inequality. Therefore $\gamma \in \mathcal{F}_{L}$. 
For the sake of simplicity we denote by $\gamma_{n}$ the subsequence $\gamma_{n_{k}}$.

We are going to show that $\delta_{\gamma_{n}}$ converges to $\delta_{\gamma}$. To this end we assume that there is given a continuous function $\varphi$, henceforth

$$
\left\langle\delta_{\gamma_{n}}, \varphi\right\rangle=\int_{\gamma_{n}} \varphi d \gamma_{n}=\int_{-\frac{1}{2}}^{\frac{1}{2}} \varphi\left(F_{n}(\ell, 0)\right)\left|F_{n}^{\prime}(\ell, 0)\right| d \ell
$$

The sequence $F_{n}$ satisfies uniformly $(5)$, using the assumption $(\mathcal{H})$ it follows that

$$
\left|F_{n}^{\prime}(\cdot, 0)\right| \rightarrow\left|F^{\prime}(\cdot, 0)\right| \quad \text { weakly }-(*) \text { in } L^{2}\left(-\frac{1}{2}, \frac{1}{2}\right) .
$$

Since $\varphi$ is continuous, hence uniformly continuous on $\bar{\Omega}_{0}$,

$$
\varphi\left(F_{n}(\cdot, 0)\right) \rightarrow \varphi(F(\cdot, 0)) \text { in } L^{\infty}\left(-\frac{1}{2}, \frac{1}{2}\right)
$$

thus

$$
\left\langle\delta_{\gamma_{n}}, \varphi\right\rangle \rightarrow \int_{-\frac{1}{2}}^{\frac{1}{2}} \varphi(F(\ell, 0))\left|F^{\prime}(\ell, 0)\right| d \ell=\left\langle\delta_{\gamma}, \varphi\right\rangle
$$

Let us consider a sequence of admissible curves $\gamma_{n}$ and the admissible curve $\gamma$ such that $\delta_{\gamma_{n}}$ converges to $\delta_{\gamma}$ weakly in $\mathcal{M}_{b}\left(\Omega_{0}\right)$. We denote by $u_{n}, u$ solutions to $\mathcal{P}_{1}$ and $\mathcal{P}_{2}$ for the boundary data $\delta_{\gamma_{n}}$ and $\delta_{\gamma}$, respectively. Using Proposition 2.2, we have immediately:

Proposition 2.5 Let $\left\{\gamma_{n}\right\}, \gamma \in \mathcal{F}_{L}$ be given, such that $\delta_{\gamma_{n}} \rightarrow \delta_{\gamma}$ weakly in $\mathcal{M}_{b}\left(\Omega_{0}\right)$. Then,

$$
u_{n} \rightarrow u \quad \text { strongly in } L^{2}\left(0, T ; L^{2}(\Sigma)\right) \text { and weakly in } L^{2}(0, T ; V)
$$

and

$$
\frac{d u_{n}}{d t} \rightarrow \frac{d u}{d t} \quad \text { weakly in } L^{2}\left(0, T ; V^{\prime}\right)
$$

$\mathrm{RR} \mathrm{n}^{\circ} 3185$ 


\subsection{The shape optimization problem}

We are interested in the following shape optimization problem:

Minimize a cost functional

$$
J(\gamma)=\left\|u_{\gamma}(x, t)-u_{d}\right\|
$$

where $u_{\gamma}$ denotes the solution to the heat equation $\mathcal{P}_{1}(\gamma)$ for any $\gamma \in \mathcal{F}_{L}$ and the Dirac measure $\delta_{\gamma}$ in the boundary conditions, $u_{d}$ is a given function, and $\|\cdot\|$ is a norm, or a seminorm on the space $W(0, T)$ which will be specified below.

Using the above results we are in position to prove an existence result for the optimization problem under considerations. Assume that there is given a functional $J(\cdot)$ continuous with respect to $u=u_{\gamma}$ in the norm topology of the space $L^{2}(0, T ; H)$ or weakly lower semicontinuous on $L^{2}(0, T ; V)$. Let us consider, as an example, the following cost functionals

$$
J_{1}(\gamma)=\int_{0}^{T} \int_{\Sigma}\left(u_{\gamma}(t, x)-u_{d}\right)^{2} d x+\int_{0}^{T} \int_{\Sigma}\left|\nabla u_{\gamma}(t, x)-\nabla u_{d}\right|^{p} d x
$$

or

$$
J_{2}(\gamma)=\int_{\Sigma}\left(u_{\gamma}(T, x)-u_{d}(x)\right)^{2} d x
$$

Theorem 2.6 There exists a solution to the minimization problems

$$
\inf _{\gamma \in \mathcal{F}_{L}} J_{1}(\gamma) \quad \text { or } \quad \inf _{\gamma \in \mathcal{F}_{L}} J_{2}(\gamma)
$$

Proof. Let $\left\{\gamma_{n}\right\}$ denote a minimizing sequence, then for a subsequence, still denoted by $\left\{\gamma_{n}\right\}$ we have, by Proposition 2.4 and 2.5,

$$
u_{\gamma_{n}} \rightarrow u_{\gamma} \quad \text { strongly in } L^{2}\left(0, T ; L^{2}(\Sigma)\right) \text { and weakly in } L^{2}\left(0, T ; H^{1}(\Sigma)\right)
$$

hence

$$
\liminf J_{1}\left(\gamma_{n}\right) \geq J_{1}(\gamma)
$$

therefore $\gamma$ is a minimum of $J_{1}$.

For $J_{2}$, we use the same argument, by Lemma 2.3 above, which completes the proof of theorem. 


\subsection{Optimality conditions}

We start with the auxiliary results on the differentiability of the following shape functional

$$
\gamma \rightarrow \int_{\gamma} \mathcal{G} d \gamma
$$

We assume that the function $\mathcal{G} \in L^{1}(\gamma)$ may depend on the curve $\gamma$. We use the material derivative method (Sokolowski and Zolesio, 1992)

Let the sufficiently smooth mapping $\mathcal{F}_{s}: \mathbb{R}^{3} \mapsto \mathbb{R}^{3}$ be given, $s \in[0, \delta)$ is a parameter, such that $F_{s}=\mathcal{F}_{s \mid Q}$ for any $s \in[0, \delta)$ satisfies the assumptions of definition 2.1, i.e.

$$
\begin{aligned}
& F_{s}(Q)=\mathcal{O} \\
& \|_{s}(I)=\gamma \\
&\left\|F_{s}\right\|_{W^{1, \infty}(Q)} \leq L_{1}
\end{aligned}
$$

Given parametrization $\left\{x_{s}(\ell), y_{s}(\ell)\right\}, \ell \in[0,1]$, of the curve $\gamma_{s}$, we denote

$$
j(s)=\int_{\gamma_{s}} \mathcal{G}_{s} d \gamma_{s}=\int_{0}^{1} \mathcal{G}_{s}\left(x_{s}(\ell), y_{s}(\ell)\right) \sqrt{x_{s}^{\prime 2}(\ell)+y_{s}^{\prime 2}(\ell)} d \ell
$$

The derivative takes the form

$$
\begin{gathered}
j^{\prime}(s)=\int_{0}^{1}\left\{\frac{\partial \mathcal{G}_{s}}{\partial s}+\nabla \mathcal{G}_{s}\left(x_{s}(\ell), y_{s}(\ell)\right) \cdot \xi_{s}(\ell)\right\} \sqrt{x_{s}^{\prime 2}(\ell)+y_{s}^{\prime 2}(\ell)} d \ell \\
+\int_{0}^{1} \mathcal{G}_{s}\left(x_{s}(\ell), y_{s}(\ell)\right) \tau_{s}(\ell) \cdot \frac{d \xi_{s}}{d \ell}(\ell) d \ell
\end{gathered}
$$

where $\tau_{s}(\ell)=\frac{\left(x_{s}^{\prime}(\ell), y_{s}^{\prime}(\ell)\right)}{\sqrt{x_{s}^{\prime 2}(\ell)+y_{s}^{\prime 2}(\ell)}}$ is the unit tangent vector to $\gamma$ and $\xi_{s}(\ell)=\frac{d}{d s}\left(x_{s}(\ell), y_{s}(\ell)\right)$.

Under regularity assumptions, after integration by parts the latter integral can be rewritten in the following form

$$
\begin{gathered}
\int_{0}^{1} \mathcal{G}_{s}\left(x_{s}, y_{s}\right) \tau_{s} \cdot \frac{d \xi_{s}}{d \ell} d \ell \\
=-\int_{0}^{1}\left\{\nabla \mathcal{G}_{s}\left(x_{s}, y_{s}\right) \cdot\left(x_{s}, y_{s}\right) \tau_{s} \cdot \xi_{s}+\mathcal{G}_{s}\left(x_{s}, y_{s}\right) \frac{d \tau_{s}}{d \ell} \cdot \xi_{s}\right\} d \ell \\
+\mathcal{G}_{s}\left(x_{s}(1), y_{s}(1)\right) \tau_{s}(1) \cdot \xi_{s}(1)-\mathcal{G}_{s}\left(x_{s}(0), y_{s}(0)\right) \tau_{s}(0) \cdot \xi_{s}(0)
\end{gathered}
$$

RR $n^{\circ} 3185$ 
On the other hand, we can use the material derivative method to obtain the same derivative $j^{\prime}(s)$. Namely, we introduce the vector field

$$
V(s, x, y, z)=\left(\frac{\partial \mathcal{F}_{s}}{\partial s} \circ \mathcal{F}_{s}^{-1}\right)(x, y, z)
$$

and assume that the support of the vector field is included in a small neighbourhood $\mathcal{O}(\gamma)$ of the curve $\gamma$ in $\mathbb{R}^{3}$. Furthermore, we assume that for $(x, y, z) \in \mathcal{O}(\gamma)$ and sufficiently small $z \in(-\varepsilon, \varepsilon), \varepsilon>0$, the field is of the following form

$$
V(s, x, y, z)=\left(\begin{array}{c}
V_{1}(s, x, y) \\
V_{1}(s, x, y) \\
0
\end{array}\right)=V(s, x, y, 0)
$$

The shape functional we consider takes the form

$$
J(\gamma)=\int_{\gamma} \mathcal{G} d \gamma
$$

With the vector field $V$ we associate the mapping

$$
T_{s}(V): \mathbb{R}^{3} \mapsto \mathbb{R}^{3},
$$

in particular, under our assumptions on the support of the field $V, \operatorname{supp} V \subset$ $\mathcal{O}(\gamma)$, it follows that $T_{s}(V) \equiv I$ on $\mathbb{R}^{3} \backslash \mathcal{O}(\gamma)$, where $I$ denotes the identity mapping.

Let us define the Eulerian semiderivative

$$
d J(\gamma ; V)=\lim _{s \downarrow 0} \frac{1}{s}\left(J\left(T_{s}(\gamma)\right)-J(\gamma)\right) .
$$

For

$$
\gamma_{s}=T_{s}(\gamma), \quad s \in[0, \delta)
$$

it follows that

$$
j^{\prime}\left(0^{+}\right)=d J(\gamma ; V)
$$

and therefore, by an application of the structure theorem for the shape gradient, we obtain

$$
d J(\gamma ; V)=\int_{0}^{1}\left\{{\frac{\partial \mathcal{G}_{s}}{\partial s}}_{\mid s=0}+\nabla \mathcal{G}(x(\ell), y(\ell)) \cdot \xi(\ell)\right\} \sqrt{x^{\prime 2}(\ell)+y^{\prime 2}(\ell)} d \ell
$$




$$
\begin{gathered}
+\int_{0}^{1} \mathcal{G}(x(\ell), y(\ell)) \frac{d \tau}{d \ell}(\ell) \cdot \xi(\ell) d \ell \\
+\mathcal{G}(x(1), y(1)) \tau\left(1^{-}\right) \cdot \xi(1)-\mathcal{G}(x(0), y(0)) \tau\left(0^{+}\right) \cdot \xi(0)
\end{gathered}
$$

since $V(s, x(\ell), y(\ell), 0)=\left(\xi_{s}(\ell), 0\right)$ for $\ell \in[0,1]$, and the vector $\tau(\ell) \in \Omega_{0}$, $\ell \in(0,1)$, is tangent to $\gamma$. If $\nu(\ell) \in \Omega_{0}, \ell \in(0,1)$, denotes the normal vector field on $\gamma$, the equivalent form of the first integral reads

$$
\begin{aligned}
& \int_{0}^{1}\left\{{\frac{\partial \mathcal{G}_{s}}{\partial s}}_{\mid s=0}+\nabla \mathcal{G}(x(\ell), y(\ell)) \cdot \xi(\ell)\right\} \sqrt{x^{\prime 2}(\ell)+y^{\prime 2}(\ell)} d \ell \\
& =\int_{0}^{1}\left\{\frac{\partial \mathcal{G}_{s}}{\partial s_{\mid s=0}}+[\nabla \mathcal{G}(x(\ell), y(\ell)) \cdot \nu(\ell)] \xi(\ell) \cdot \nu(\ell)\right\} \sqrt{x^{\prime 2}(\ell)+y^{\prime 2}(\ell)} d \ell
\end{aligned}
$$

since the integral part of $d J(\gamma ; V)$, by the structure theorem, depends only on the normal component $V(0, x(\ell), y(\ell), 0) \cdot n=\xi(\ell) \cdot \nu(\ell), \ell \in(0,1)$, of the field $V(0, x(\ell), y(\ell), 0)$. We denote

$$
\begin{aligned}
\int_{\gamma} \dot{\mathcal{G}} d \gamma= & \int_{0}^{1}\left\{\frac{\partial \mathcal{G}_{s}}{\partial s_{\mid s=0}}+\nabla \mathcal{G}(x(\ell), y(\ell)) \cdot \xi(\ell)\right\} \sqrt{x^{\prime 2}(\ell)+y^{\prime 2}(\ell)} d \ell \\
& \int_{\gamma} \mathcal{G} \tau^{\prime} \cdot V d \gamma=\int_{0}^{1} \mathcal{G}(x(\ell), y(\ell)) \frac{d \tau}{d \ell}(\ell) \cdot \xi(\ell) d \ell \\
& (x(1), y(1))=\left(x_{1}, y_{1}\right), \quad(x(0), y(0))=\left(x_{0}, y_{0}\right)
\end{aligned}
$$

Proposition 2.7 The shape funtional $J(\gamma)=\int_{\gamma} \mathcal{G} d \gamma$ is shape differentiable, the Eulerian semiderivative takes the following form

$$
\begin{gathered}
d J(\gamma ; V)=\int_{\gamma} \dot{\mathcal{G}} d \gamma+\int_{\gamma} \mathcal{G} \tau^{\prime} \cdot V d \gamma+\mathcal{G}\left(x_{1}, y_{1}\right) \tau\left(x_{1}^{-}, y_{1}^{-}\right) \cdot V\left(0, x_{1}, y_{1}, 0\right) \\
-\mathcal{G}\left(x_{0}, y_{0}\right) \tau\left(x_{0}^{+}, y_{0}^{+}\right) \cdot V\left(0, x_{0}, y_{0}, 0\right)
\end{gathered}
$$

where $\dot{\mathcal{G}}$ denotes the material derivative of $\mathcal{G}$ in the direction of the vector field $V$.

Now, we are in the position to obtain the shape differentiability of solutions to the problem $\mathcal{P}_{1}(\gamma)$.

RR $n^{\circ} 3185$ 
We denote $\Sigma_{s}=T_{s}(\Sigma), u_{s} \in L^{2}\left(0, T ; W^{1, p}\left(\Sigma_{s}\right)\right)$ the unique solution to the following integral identity, $u_{s}(0)=u_{0}$ in $\Sigma_{s}$,

$$
\begin{aligned}
\frac{d}{d t} \int_{\Sigma_{s}} u_{s}(t) \varphi d \Sigma_{s} & +\int_{\Sigma_{s}} \nabla u_{s}(t) \cdot \nabla \varphi d \Sigma_{s}+\int_{\Omega_{0}^{s}} u_{s}(t) \varphi d \sigma_{s}+\int_{\Omega_{1}^{s}} u_{s}(t) \varphi d \sigma_{s} \\
& =\int_{\Omega_{0}^{s}} u_{0} \varphi d \sigma_{s}+\int_{\Omega_{1}^{s}} u_{1} \varphi d \sigma_{s}+\int_{\gamma_{s}} \varphi d \gamma_{s}
\end{aligned}
$$

for all $\varphi \in W^{1, q}\left(\Sigma_{s}\right)$, where $\Omega_{i}^{s}=T_{s}\left(\Omega_{i}\right), i=0,1, \gamma_{s}=T_{s}(\gamma)$. The initial condition for $u_{s}$ makes sense since $u_{s} \in W_{s}(0, T)$, the space $W_{s}(0, T)$ is defined in the same way as $W(0, T)$ with the set $\Sigma$ replaced by $\Sigma_{s}$.

The integral identity is transported to the fixed domain $\Sigma$, so we denote $u^{s}=u_{s} \circ T_{s} \in W^{1, p}(\Sigma)$, set $\varphi=v \circ T_{s}^{-1}$, and by standard change of variables it follows that $u^{s}$ is the unique solution to the following inegral identity, $u^{s}(0)=$ $u_{0} \circ T_{s}$ in $\Sigma$,

$$
\begin{gathered}
\frac{d}{d t} \int_{\Sigma} u^{s}(t) v \operatorname{det}\left(D T_{s}\right) d \Sigma+\int_{\Sigma}\left\langle A_{s} \cdot \nabla u^{s}(t), \nabla v\right\rangle_{\mathbb{R}^{3}} d \Sigma+\int_{\Omega_{0}} u^{s}(t) v \omega_{s} d \sigma \\
+\int_{\Omega_{1}} u^{s}(t) v \omega_{s} d \sigma=\int_{\Omega_{0}} u_{0}^{s} v \omega_{s} d \sigma+\int_{\Omega_{1}} u_{1}^{s} v \omega_{s} d \sigma+\int_{\gamma} v \rho_{s} d \gamma
\end{gathered}
$$

for all $v \in W^{1, q}(\Sigma)$, where the matrix $A_{s}$, the boundary terms $\omega_{s}, \rho_{s}$ are given, sufficiently smooth functions of space variables and $s \in[0, \delta)$,

$$
\begin{aligned}
A_{s} & =\operatorname{det}\left(D T_{s}\right) D T_{s}^{-1} \cdot{ }^{*} D T_{s}^{-1} \\
\omega_{s} & =\left\|\operatorname{det}\left(D T_{s}\right)^{*} D T_{s}^{-1} \cdot n\right\|_{\mathbb{R}^{3}} \\
\rho_{s} & =\left(\frac{x_{s}^{\prime 2}(\ell)+y_{s}^{\prime 2}(\ell)}{x^{\prime 2}(\ell)+y^{\prime 2}(\ell)}\right)^{\frac{1}{2}},(x(\ell), y(\ell)) \in \gamma, \gamma_{s}=T_{s}(\gamma), \ell \in(0,1) .
\end{aligned}
$$

By an application of the implicit function theorem for solutions of the latter integral identity we obtain the existence of the weak material derivative in $W(0, T)$ and $L^{2}\left(0, T ; W^{1, p}(\Sigma)\right), \frac{6}{5}<p<\frac{3}{2}$,

$$
\dot{u}=\lim _{s \downarrow 0} \frac{1}{s}\left(u^{s}-u\right) \text {. }
$$


The material derivative $\dot{u} \in W^{1, p}(\Sigma)$ satisfies the following integral identity, $\dot{u}(0)=\dot{u}_{0}=\nabla u_{0} \cdot V(0)$ in $\Sigma$,

$$
\begin{aligned}
& \frac{d}{d t} \int_{\Sigma} \dot{u} v \operatorname{div} V(0) d \Sigma+\int_{\Sigma} \nabla \dot{u} \cdot \nabla v d \Sigma+\int_{\Sigma}\left\langle A^{\prime}(0) \cdot \nabla u, \nabla v\right\rangle_{\mathbb{R}^{3}} d \Sigma \\
& \quad+\int_{\Omega_{0}} \dot{u} v d \sigma+\int_{\Omega_{0}} u v \omega^{\prime}(0) d \sigma+\int_{\Omega_{1}} \dot{u} v d \sigma+\int_{\Omega_{1}} u v \omega^{\prime}(0) d \sigma \\
& =\int_{\Omega_{0}}\left(\dot{u}_{0}+u_{0} \omega^{\prime}(0)\right) v d \sigma+\int_{\Omega_{1}}\left(\dot{u}_{1}+u_{1} \omega^{\prime}(0)\right) v d \sigma+\int_{\gamma} v \rho^{\prime}(0) d \gamma,
\end{aligned}
$$

where we denote

$$
\begin{aligned}
A^{\prime}(0) & =\operatorname{div} V(0) I-D V(0)-{ }^{*} D V(0) \\
\omega^{\prime}(0) & =\operatorname{div} V(0)-\langle D V(0) \cdot n, n\rangle_{\mathbb{R}^{3}} \\
\rho^{\prime}(0) & =\tau \cdot D V(0) \cdot \tau
\end{aligned}
$$

Let us consider for example the shape functional

$$
J(\gamma)=\int_{0}^{T} \int_{\Sigma}\left(u_{\gamma}(t, x)-u_{d}(t, x)\right)^{2} d \Sigma d t
$$

Theorem 2.8 A solution to the minimization problem

$$
\inf _{\gamma \in \mathcal{F}_{L}} J(\gamma)
$$

satisfies the first order necessary optimality conditions

$$
d J(\gamma ; V)=0
$$

for all admissible vector fields $V$, where

$$
d J(\gamma ; V)=2 \int_{0}^{T} \int_{\Sigma}\left(u(\gamma)-u_{d}\right) \dot{u} d \Sigma+\int_{0}^{T} \int_{\Sigma}\left|u(\gamma)-u_{d}\right|^{2} \operatorname{div} V d \Sigma
$$

The optimality conditions can be further simplfied using the standard adjoint state equation.

RR $n^{\circ} 3185$ 


\section{Behaviour of the optimal solution when $T$ goes to $+\infty$}

In the paper (Henrot, Horn and Sokolowski, 1996) we investigated the stationary problem, namely

$(\mathcal{S P}(\gamma)) \quad\left\{\begin{aligned}-\Delta U_{\gamma} & =0 \quad \text { in } \Omega \\ \frac{\partial U_{\gamma}}{\partial n} & =0 \quad \text { on } \Gamma \\ -\frac{\partial U_{\gamma}}{\partial n} & =U_{\gamma}-\varphi_{1} \quad \text { on } \Omega_{1} \\ -\frac{\partial U_{\gamma}}{\partial n} & =U_{\gamma}-\varphi_{0}-\delta_{\gamma} \quad \text { on } \Omega_{0} .\end{aligned}\right.$

We proved, in particular, that functionals analogous to those given by (12) (without dependance in time), have a minimum in the class of admissible curves $\mathcal{F}_{L}$.

In this section, we are interested in the behaviour of the optimal curve that we have obtained for a time interval $[0, T]$, when $T$ goes to infinity. More precisely, we would like to prove that this optimal solution, say $\gamma_{T}$ (since it depends on $T$ ), converges to the optimal solution for the stationary case. Of course, we are going to work in this section with the following functionals:

$$
J_{T}(\gamma):=\int_{\Sigma}\left(u_{\gamma}(T, x)-u_{d}(x)\right)^{2} d x=\left\|u_{\gamma}(T)-u_{d}\right\|_{L^{2}(\Sigma)}
$$

and

$$
J_{\infty}(\gamma):=\int_{\Sigma}\left(U_{\gamma}(x)-u_{d}(x)\right)^{2} d x=\left\|U_{\gamma}-u_{d}\right\|_{L^{2}(\Sigma)} .
$$

where $u_{\gamma}$ is the solution of the evolutionary problem $\mathcal{P}_{1}(\gamma)$, and $U_{\gamma}$ the solution of the stationary problem $\mathcal{S P}(\gamma)$. Then, we have

Theorem 3.1 Let us denote by $\gamma_{T}$ (resp. $\gamma_{\infty}$ ) an optimal curve for the functional $J_{T}$ (resp. $J_{\infty}$ ) defined above. Then $\gamma_{T}$ converges uniformly to $\gamma_{\infty}$, up to a subsequence, in the sense that the parametrizations $F_{T}$ given by the definition 1 converges uniformly to $F_{\infty}$ when $T \rightarrow+\infty$. 
First of all, let us recall a classical convergence result.

Lemma 3.2 Let $\gamma \in \mathcal{F}_{L}$ be fixed. Then $u_{\gamma}(x, T)$ converge to $U_{\gamma}(x)$ strongly in $L^{2}(\Sigma)$ when $T \rightarrow+\infty$.

Proof. We use the spectral representation of the solution of the problem $\mathcal{P}_{1}(\gamma)$. Let us denote by $\lambda_{1}<\lambda_{2} \leq \ldots \lambda_{n} \leq \ldots$ and $\varphi_{1}, \varphi_{2}, \ldots, \varphi_{n}, \ldots$ the sequence of eigenvalues and eigenfunctions for the Neumann-Robin problem

$$
\left\{\begin{aligned}
-\Delta \varphi & =\lambda \varphi & & \text { in } \Omega, \\
\frac{\partial \varphi}{\partial n} & =0 & & \text { on } \Gamma, \\
-\frac{\partial \varphi}{\partial n} & =\varphi & & \text { on } \Omega_{1} \cup \Omega_{0} .
\end{aligned}\right.
$$

Since the first eigenvalue of this problem is also given by

$$
\lambda_{1}=\inf _{v \in H^{1}(\Sigma)} \frac{\int_{\Sigma}|\nabla v|^{2} d x+\int_{\Omega_{0} \cup \Omega_{1}} v^{2} d x}{\int_{\Sigma} v^{2} d x}
$$

it is strictly positive by the Poincaré-Friedrichs inequality.

Now, since the function $U(t, x):=U_{\gamma}(x)$ (independant of time) is solution of the following problem

$$
\left\{\begin{array}{cccc}
\frac{\partial U}{\partial t}-\Delta U & = & 0 & \text { in } \Sigma \times(0, T), \\
\frac{\partial U}{\partial n} & = & 0 & \text { on } \Gamma \times(0, T), \\
-\frac{\partial U}{\partial n} & = & U-\varphi_{1} & \text { on } \Omega_{1} \times(0, T), \\
-\frac{\partial U}{\partial n} & = & U-\varphi_{0}-\delta_{\gamma} & \text { on } \Omega_{0} \times(0, T) \\
U(x, 0) & = & U_{\gamma}(x) & \text { in } \Sigma,
\end{array}\right.
$$

RR $n^{\circ} 3185$ 
the difference $v(x, t)=u_{\gamma}(x, t)-U(x, t)$ is solution of

$$
\left\{\begin{array}{rrrr}
\frac{\partial v}{\partial t}-\Delta v & = & 0 & \text { in } \Sigma \times(0, T), \\
\frac{\partial v}{\partial n} & = & 0 & \text { on } \Gamma \times(0, T), \\
-\frac{\partial v}{\partial n} & = & v & \text { on }\left(\Omega_{0} \cup \Omega_{1}\right) \times(0, T), \\
v(x, 0) & = & u_{0}(x)-U_{\gamma}(x):=v_{0}(x) & \text { in } \Sigma .
\end{array}\right.
$$

Therefore, it can be expanded in the basis of eigenfunctions:

$$
v(x, t)=\sum_{k=1}^{\infty} v_{k} e^{-\lambda_{k} t} \varphi_{k}(x)
$$

where the coefficients $v_{k}$ are given by the expansion of the initial data $v_{0}(x)=$ $\sum_{k=1}^{\infty} v_{k} \varphi_{k}(x)$. So, it is clear by the Parseval identity that

$$
\|v(x, T)\|_{L^{2}(\Sigma)}^{2} \leq e^{-2 \lambda_{1} T}\left\|v_{0}(x)\right\|_{L^{2}(\Sigma)}^{2} \longrightarrow 0
$$

when $T \rightarrow \infty$, which completes the proof of the lemma.

Proof. (of theorem 3.1) Theorem 2.6 shows that for each fixed $T>0$, there exists (at least) one optimal curve, say $\gamma_{T} \in \mathcal{F}_{L}$ which minimizes the functional $J_{T}$ defined by (15). According to proposition 2.1, there exists a curve $\gamma^{\star} \in \mathcal{F}_{L}$ and a subsequence $\gamma_{T_{n}}$ which converge uniformly to $\gamma^{\star}$ in the sense defined in the theorem, and such that

$$
\delta_{\gamma_{T_{n}}} \rightarrow \delta_{\gamma^{\star}} \quad \text { weak- }(*) \text { in the space }\left(W^{1, p}(\Sigma)\right)^{\prime},
$$

in fact for $p>2$, for $p=2$ the result is in general false. In order to prove the theorem, it is sufficient to prove the following lemma.

Lemma 3.3 The sequence $u_{\gamma_{T_{n}}}\left(x, T_{n}\right)$ converges weakly to $u_{\gamma^{\star}}(x)$ in $L^{2}(\Sigma)$ when $n \rightarrow \infty$.

We continue the proof of theorem 2.6, the proof of lemma 3.3 is given below. Using the optimal solutions for $T_{n}$, we have

$$
\int_{\Sigma}\left(u_{\gamma_{T_{n}}}\left(x, T_{n}\right)-u_{d}(x)\right)^{2} d x \leq \int_{\Sigma}\left(u_{\gamma_{\infty}}\left(x, T_{n}\right)-u_{d}(x)\right)^{2} d x .
$$


Now, lemma 2.6 shows that $u_{\gamma_{\infty}}\left(x, T_{n}\right)$ converges strongly to $U_{\gamma_{\infty}}(x)$ when $n \rightarrow \infty$, then by lower semi-continuity of the norm and in view of lemma 3.3, we have

$$
\begin{aligned}
J_{\infty}\left(\gamma^{\star}\right)=\int_{\Sigma}\left(u_{\gamma^{\star}}(x)-u_{d}(x)\right)^{2} d x & \leq \liminf \int_{\Sigma}\left(u_{\gamma_{T_{n}}}\left(x, T_{n}\right)-u_{d}(x)\right)^{2} d x \\
\leq \lim \int_{\Sigma}\left(u_{\gamma_{\infty}}\left(x, T_{n}\right)-u_{d}(x)\right)^{2} & =\int_{\Sigma}\left(U_{\gamma_{\infty}}(x)-u_{d}(x)\right)^{2}=\inf J_{\infty}
\end{aligned}
$$

which proves that $\gamma^{\star}$ is also a minimum of the functional $J_{\infty}$ which completes the proof of the theorem 2.6.

Proof. (of lemma 3.3) Let $\varepsilon>0$ be fixed. According to the proposition 2.4 of the stationary problem ( $\mathrm{cf}$ Henrot, Horn and Sokolowski, 1996), the sequence $U_{\gamma_{T_{n}}}$ converges strongly in $L^{2}(\Sigma)$ to $U_{\gamma^{\star}}$, in particular, it is bounded,

$$
\left\|U_{\gamma_{T_{n}}}\right\|_{L^{2}} \leq M \quad \text { and } \quad\left\|U_{\gamma^{\star}}\right\|_{L^{2}} \leq M
$$

Let us fix a positive number $\tau$ large enough such that

$$
e^{-\lambda_{1} \tau}\left(\left\|u_{0}\right\|_{L^{2}}+M\right) \leq \varepsilon .
$$

Let $v$ be a fixed function in $L^{2}(\Sigma)$, we have to prove that

$$
\int_{\Sigma}\left(u_{\gamma_{T_{n}}}\left(x, T_{n}\right)-u_{\gamma^{\star}}(x)\right) v(x) d x \longrightarrow 0 .
$$

Let us write the left member of (20) as

$$
\begin{aligned}
& \int_{\Sigma}\left(u_{\gamma_{T_{n}}}\left(x, T_{n}\right)-u_{\gamma_{T_{n}}}(x, \tau)\right) v(x) d x+\int_{\Sigma}\left(u_{\gamma_{T_{n}}}(x, \tau)-u_{\gamma^{\star}}(x, \tau)\right) v(x) d x+ \\
& \quad+\int_{\Sigma}\left(u_{\gamma^{\star}}(x, \tau)-u_{\gamma^{\star}}(x)\right) v(x) d x
\end{aligned}
$$

It is easy to let the second term in (21) less than $\varepsilon$ for $n$ large enough using the weak convergence of $u_{\gamma_{T_{n}}}(x, \tau)$ to $u_{\gamma^{\star}}(x, \tau)$ (cf Lemma 1). Moreover, thanks to (19) and the proof of the Lemma 2 , we have

$$
\left\|u_{\gamma^{\star}}(x, \tau)-U_{\gamma^{\star}}(x)\right\|_{L^{2}} \leq e^{-\lambda_{1} \tau}\left\|u_{0}-U_{\gamma^{\star}}\right\|_{L^{2}} \leq \varepsilon,
$$

so the third term in $(21)$ is also estimated from above by $\varepsilon\|v\|$. It remains to look at the first term. Using one more time the spectral expansion of the solution of the parabolic problem, we are able to write

$$
u_{\gamma_{T_{n}}}\left(x, T_{n}\right)-u_{\gamma_{T_{n}}}(x, \tau)=\sum_{k=1}^{\infty} v_{k}\left(e^{-\lambda_{k} T_{n}}-e^{-\lambda_{k} \tau}\right) \varphi_{k}(x) .
$$

RR $n^{\circ} 3185$ 
Therefore, by Parseval identity

$$
\left\|u_{\gamma_{T_{n}}}\left(x, T_{n}\right)-u_{\gamma_{T_{n}}}(x, \tau)\right\|_{L^{2}}^{2}=\sum_{k=1}^{\infty} v_{k}^{2}\left(e^{-\lambda_{k} T_{n}}-e^{-\lambda_{k} \tau}\right)^{2} .
$$

Now, for $T_{n}>\tau$, we have

$$
0<e^{-\lambda_{k} \tau}-e^{-\lambda_{k} T_{n}}<e^{-\lambda_{k} \tau}<e^{-\lambda_{1} \tau}
$$

then

$$
\left\|u_{\gamma_{T_{n}}}\left(x, T_{n}\right)-u_{\gamma_{T_{n}}}(x, \tau)\right\|_{L^{2}}^{2} \leq e^{-2 \lambda_{1} \tau} \sum_{k=1}^{\infty} v_{k}^{2}=e^{-2 \lambda_{1} \tau}\left\|u_{0}-U_{\gamma_{T_{n}}}\right\|_{L^{2}}^{2} \leq \varepsilon^{2} .
$$

the last inequality coming from (18) and (19). Then the lemma is proved.

\section{References}

[1] Adams R.A. (1975): Sobolev Spaces. — Academic Press, New York.

[2] Aubin, J.P. (1963): Un théorème de compacité, C.R.Acad.Sci., 256, pp. 5042-5044.

[3] Bucur, D. and Zolesio, J.P. (1995): N-dimensional shape optimization under capacitary constraints, J. Diff. Eq., 123-2, pp. 504-522.

[4] Daniliuk, I.I. (1975): Nonsmooth boundary value problems in the plane. Nauka, Moscou (in Russian).

[5] Henrot, A., Horn, W. and Sokołowski, J. (1996): Domain optimization problem for stationary heat equation, Appl. Math. and Comp. Sci., vol.6, No.2, pp. 1-21.

[6] Hoffmann, K.-H. and Sokołowski, J.(1994): Interface optimization problems for parabolic equations. - Control and Cybernetics, v. 23, pp. 445-452.

[7] Lions J.L., Magenes E. (1968): Problèmes aux limités non homogènes. — Dunod, Paris. 
[8] Sokołowski, J. and Zolesio, J.-P.(1992): Introduction to Shape Optimization. Shape sensitivity analysis. - Springer Verlag, New York.

[9] Ziemer P.W. (1989): Weakly Differentiable Functions. — Springer Verlag, New York.

RR $n^{\circ} 3185$ 
Unit`e de recherche INRIA Lorraine, Technop^ole de Nancy-Brabois, Campus scientifique, 615 rue du Jardin Botanique, BP 101, 54600 VILLERS LÈS NANCY

Unit'e de recherche INRIA Rennes, Irisa, Campus universitaire de Beaulieu, 35042 RENNES Cedex

Unit'e de recherche INRIA Rh^one-Alpes, 655, avenue de l'Europe, 38330 MONTBONNOT ST MARTIN

Unit'e de recherche INRIA Rocquencourt, Domaine de Voluceau, Rocquencourt, BP 105, 78153 LE CHESNAY Cedex

Unit’e de recherche INRIA Sophia-Antipolis, 2004 route des Lucioles, BP 93, 06902 SOPHIA-ANTIPOLIS Cedex

Éditeur

INRIA, Domaine de Voluceau, Rocquencourt, BP 105, 78153 LE CHESNAY Cedex (France)

ISSN 0249-6399 